\title{
ISCHAEMIC STROKE IN YOUNG WOMAN DUE TO PROTEIN-C DEFICIENCY
}

\author{
Selvaraj Pitchai ${ }^{1}$, Ramu$^{2}$, Mithun Mathiyazhakan³, Sravankumar Sampati4
}

1 Professor, Department of General Medicine, Sri Venkateswaraa Medical College Hospital and Research Centre, Pudhucherry. 2Professor, Department of Neurology, Chennai Medical Hospital and Research Centre, Trichy.

${ }^{3}$ Postgraduate Resident, Department of General Medicine, Sri Venkateswaraa Medical College Hospital and Research Centre, Pudhucherry.

${ }^{4}$ Postgraduate Resident, Department of General Medicine, Sri Venkateswaraa Medical College Hospital and Research Centre, Pudhucherry.

HOW TO CITE THIS ARTICLE: Pitchai S, Ramu, Mathiyazhakan M, et al. Ischaemic stroke in young woman due to protein-C deficiency. J. Evolution Med. Dent. Sci. 2017;6(73):5278-5280, DOI: 10.14260/Jemds/2017/1146

\section{PRESENTATION OF CASE}

A 30-year-old previously healthy female patient was admitted in this teaching hospital with history of sudden onset of sensory loss and weakness of left upper and lower limbs. She had headache for 10 days before the onset of weakness. There had been no loss of consciousness, seizures, chest pain or giddiness. She did not have any bleeding diathesis in the past. No history of Diabetes Mellitus, Hypertension or TIA. She had no history of neurological dysfunction in the past. No family history of thromboembolic stroke or CAD. Parents married out of second degree consanguineous marriage and both are alive, and she had five brothers of which one was born dead. Her 10year-old son is healthy, but his protein-C level was also low $55.3(70-140)$. Other close relatives were not available for investigations.

\section{Examination}

Examination of the patient showed weakness of left upper and lower limbs with 3 - 4/5 power and brisk reflexes and plantar extensor on left side. Cerebellar functions, sphincters and other neurological examinations were largely normal. Carotid on the right side was feeble but no bruit. There was diminished sensation on the left half of the body. Investigations like ECG, chest $\mathrm{x}$-ray, echocardiogram, immunological studies like AntiDS DNA antibody, ANA were normal. CBC, LFT, serum electrolytes, sugar, urea, creatinine, urine analysis and platelet counts were normal. Lipid profile showed slight elevation of triglycerides. Blood VDRL and HIV were negative. Serum fibrinogen, prothrombin time, PTT, BT and CT was normal. Serum protein-S was normal 76.9 (60 - 140).

Protein-C was very much reduced (22.9), normal being (70 - 140). The absolute concentration of protein-C and antiphospholipid antibodies and antithrombin-III were not measured. CT scan brain was normal. MRI brain showed small acute pontine infarct on ventral pons (posterior circulation stroke) on the right side. MR-Angio showed hypoplastic right vertebral artery and right anterior communicating artery. MRV was normal. Carotid and vertebral arteries Doppler showed mild atherosclerotic changes with soft plaques in right proximal internal carotid artery, but no thrombosis

Financial or Other, Competing Interest: None.

Submission 25-05-2017, Peer Review 26-08-2017,

Acceptance 01-09-2017, Published 11-09-2017.

Corresponding Author:

Dr. Selvaraj Pitchai,

Professor, Department of General Medicine,

Sri Venkateswaraa Medical College

Hospital and Research Centre,

Pudhucherry.

E-mail: Selvarajaps@rediffmail.com

DOI: $10.14260 /$ jemds/2017/1146 (c) $(1)(5)$

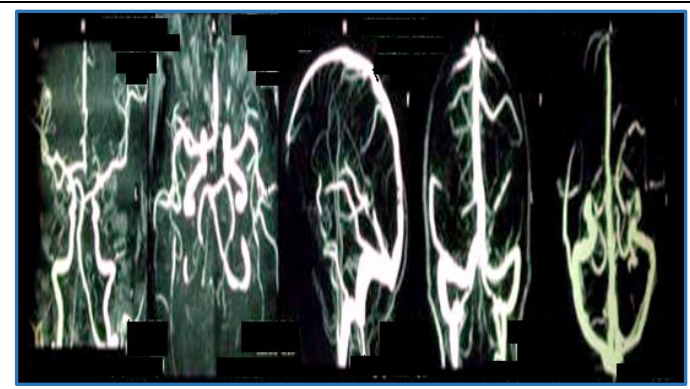

Figure 1. MR Angio showing Hypoplastic Right Vertebral Artery and Right Anterior Communicating Artery

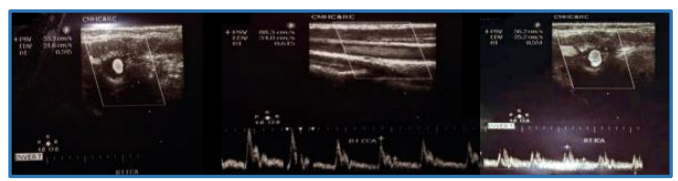

Figure 2. Doppler Studies of Right Carotid Artery
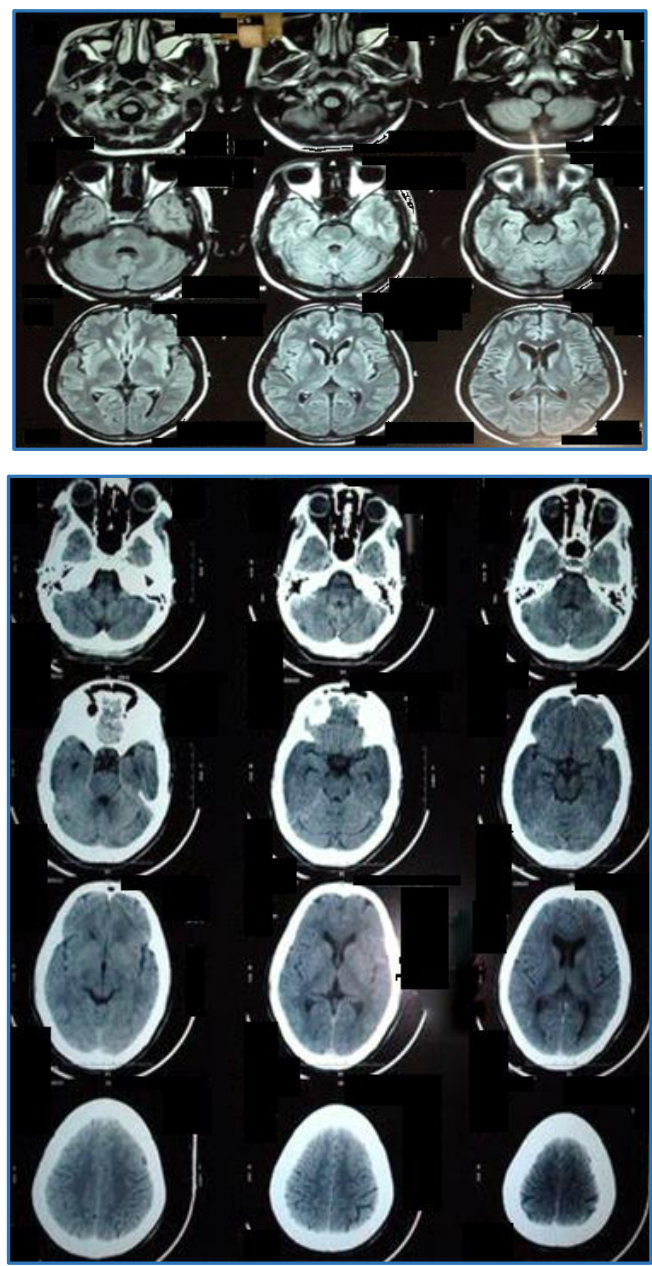

Figure 3. Patient, 30/F. CT-Brain-Normal 


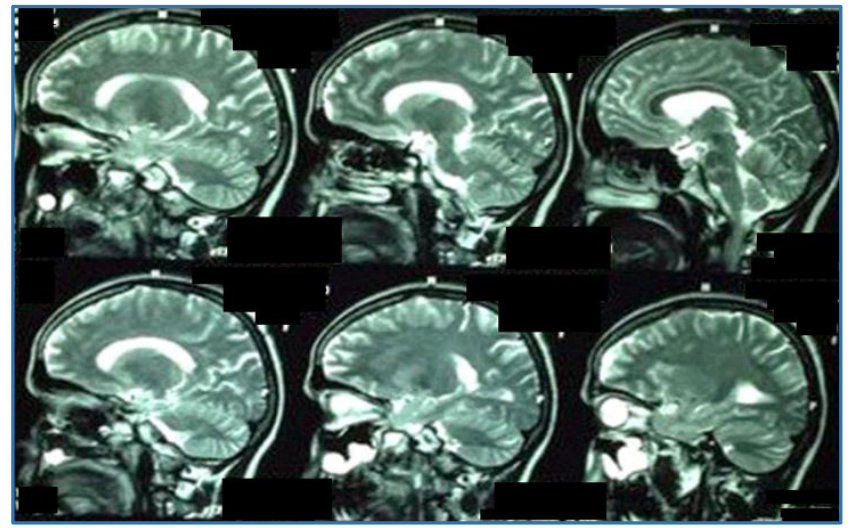

Figure 4. MRI-Brain showing Acute Non-Haemorrhagic Pontine Infarct (Posterior Circulation Stroke) in Ventral Pons Right Side

\section{DIFFERENTIAL DIAGNOSIS}

The following conditions should be considered as differential diagnosis of stroke in young.

\section{Cardiac (35.4\%)}

- Mural thrombus.

- Mitral valve prolapse.

- Aortic valve defect.

- Rheumatic heart disease.

- Congenital heart disease.

\section{Haematologic (17\%)}

- Oral contraceptives.

- Lupus anticoagulant.

- Pregnancy.

- Alcohol.

Non-Atherosclerotic Vascular Disease (16.7\%)

- Postoperative thromboembolus.

- Post-traumatic arterial thrombus.

- Carotid artery dissection.

- Vasculitis.

- $\quad$ Migraine.

- Unknown.

\section{CLINICAL DIAGNOSIS}

Cerebrovascular accident-ischaemic stroke in young with left hemiplegia and hemianaesthesia.

\section{PATHOLOGICAL DISCUSSION}

The pathology of lacunar infarcts may include microatheroma, microembolism, hypoperfusion due to microatheroma, fibrinoid necrosis or lipohyalinosis of penetrating arteries resulting in lacunar infarct. There are innumerable causes for lacunar infarcts. The protein-C level may fall following ischaemic stroke due to consumption, but in our patient subsequent protein-C estimation was also low several days after stroke.

The coagulopathy in protein- $C$ deficiency is caused by impaired inactivation of factors Va and VIIIa by activated protein-C after the propagation phase of coagulation activation. Long-term management in severe protein-C deficiency involves anticoagulation with or without a protein$C$ replacement regimen. Severe protein-C deficiency can be managed by protein- $\mathrm{C}$ replacement in the form of fresh frozen plasma (FFP) or human plasma derived viral inactivated protein-C concentrate.

\section{DISCUSSION OF MANAGEMENT}

The patient was treated with parenteral anticoagulants initially followed by oral anticoagulants and statins. Patient quickly improved with good power and she was discharged with statins and oral anticoagulants.

Protein-C deficiency mainly causes venous thrombosis. The incidence is 1 in 20000. Asymptomatic protein- $C$ deficiency may be 1 in 200 - 300. So far only few cases of arterial thrombosis have been reported and majority of them are acute MI followed by ischaemic stroke and peripheral arterial thrombosis. Protein-C deficiency increases the risk of stroke or MI when it is associated with other risk factors like smoking, alcohol, dyslipidaemia and hypercoagulable states than by itself. It should be considered as the cause, particularly when young people develop stroke or the cause is unclear. We have seen two cases so far and both of them are around 30 years of age in this institution- one case was admitted with pure dysphasia and another case presented with aphasia with right upper limb weakness.

Even though it occurs as autosomal dominant or rarely as recessive, sporadic cases of protein-C deficiency with arterial thrombosis have also been reported (Dusser et al). ${ }^{1}$ In some case protein-C deficiency has produced temporary blindness (Amaurosis fugax) (Smith and Ens et al). ${ }^{2}$ Kemkes-Matthes et al reported a case of carotid artery occlusion due to protein-C deficiency. Seshia and Israels et al have described a case of acute hemiparesis in a 17-year girl due to protein-C deficiency, but CT-brain was normal in that patient. ${ }^{3}$ Low levels of protein-C were first associated with venous thrombosis, (Griffin et al in 1982).

In our patient, the sudden onset of left-sided hemiparesis and hemianaesthesia was probably due to a lacunar infarct in the ventral pons (posterior circulation stroke), as evidenced by MRI. In many cases, the lacunar infarct in brain resulting from microembolism from carotid or MCA are not picked up by either CT or MRI scan in the initial stages. In about $30 \%$ $40 \%$ of embolic strokes, the source of embolism may not be determined (cryptogenic stroke) even though the patient may be presenting with neurological deficit. In addition, MRA in this patient showed hypoplastic right vertebral artery and hypoplastic left anterior communicating artery in this patient, but MRV was normal.

Posterior circulation stroke usually causes crossed hemiplegia (peduncular hemiplegia), but lacunar infarcts in the brainstem may produce pure motor hemiparesis or sensory stroke or both. Lacunar infarcts are caused by occlusion of small penetrating branches in internal capsule, basal ganglia, pons, thalamus, corona radiate or cerebral peduncle. Lacunar syndrome can result in pure motor or sensory stroke, ataxic hemiparesis, isolated dysphasia, dysarthria clumsy hand syndrome. The term pure motor hemiparesis or pure sensory stroke due to lacunar infarct was first described in 1965 by Fisher and Curry. Lacunar syndrome can be total or partial. The partial motor deficit includes isolated brachial monoplegia, unilateral vocal cord palsy and brachiofacial palsy. Lacunar infarcts generally carry good prognosis, but multiple lacunar infarcts can cause dementia. CT brain or MRI are best diagnostic tools to diagnose lacunar infarcts. Sometimes lacunar infarcts may be missed by both CT 
and MRI, hence CT or MRI should be repeated few days later since enhancement of lesions occurs after few days.

Lacunar infarction is more common in carotid territory than vertebrobasilar territory in the ratio of 70:30. Atypical lacunar syndromes such as isolated $3^{\text {rd }}$ nerve palsy, foville's syndrome, isolated eye and other movement disorders like chorea, hemiballismus, dystonia, parkinsonism, aphasia, visual or memory impairment have also been reported. We have also seen one isolated case of dysarthria clumsy hand syndrome and 1 case of isolated dysphasia in the last 3 months in our hospital.

\section{Conclusion}

Here, we are presenting a rare case of left-sided pure motor hemiparesis and hemianaesthesia of posterior circulation stroke in ventral pons due to protein-C deficiency associated with dyslipidaemia. Besides, the patient's 10-year-old son also had low level of protein-C (55.3), thereby strongly emphasising a genetic trait in this case. ${ }^{4}$ Hence, we conclude that protein-C deficiency associated with dyslipidaemia probably might have caused posterior circulation stroke in this patient, involving one of the paramedian branches of basilar artery in ventral pons, even though other unknown factors may have played a role. In conclusion, therefore, it is important to recognise protein-C deficiency as a significant risk factor in young patients presenting with ischaemic stroke or myocardial infarction of unknown cause. ${ }^{5}$ However, the study of protein-C deficiency causing CAD or CVA must involve a large population in multicentres. This case is presented for its rarity namely- 1 . A young woman presenting with posterior circulation stroke due to protein-C deficiency; 2 . Her son also had asymptomatic low protein-C level; 3. Posterior circulation stroke due to arterial thrombosis; 4. Hypoperfusion due to hypoplastic right vertebral artery and hypoplastic right anterior communicating artery (haemodynamic theory).

\section{FINAL DIAGNOSIS}

Cerebrovascular accident- acute non-haemorrhagic pontine infarct (posterior circulation stroke) in ventral pons right side due to protein-C deficiency.

\section{REFERENCES}

[1] Dusser A, Boyer-neumann C, Wolf M. Temporary protein $\mathrm{C}$ deficiency associated with cerebral arterial thrombosis in childhood. J pediatr 1988;113:849-51.

[2] Smith DB, Ens GE. Protein deficiency: a cause of amaurosis fugax. J Neurol Neurosurg psychiatry 1987;50(3):361-2.

[3] Israels SJ, Seshia SS. Childhood stroke associated with protein C or S deficiency. J Pediatr 1987;111(4):562-4.

[4] Milectich J, Sherman L, Broze G. Absence of thrombosis in subjects with heterozygous protein $\mathrm{C}$ deficiency. $\mathrm{N}$ Engl J Med 1987;317(16):991-6.

[5] Hacker SM, Williamson BD, Lisco S, et al. Protein C deficiency and acute myocardial infarction in the third decade. Am J Cardiol 1991;68(1):137-8. 